\title{
Multiparametric Profiling of Single Nanoscale Extracellular Vesicles by Combined Atomic Force and Fluorescence Microscopy: Correlation and Heterogeneity in Their Molecular and Biophysical Features
}

\author{
Sara Cavallaro,* Federico Pevere, Fredrik Stridfeldt, André Görgens, Carolina Paba, \\ Siddharth S. Sahu, Doste R. Mamand, Dhanu Gupta, Samir El Andaloussi, Jan Linnros, \\ and Apurba Dev**
}

Being a key player in intercellular communications, nanoscale extracellular vesicles (EVs) offer unique opportunities for both diagnostics and therapeutics. However, their cellular origin and functional identity remain elusive due to the high heterogeneity in their molecular and physical features. Here, for the first time, multiple EV parameters involving membrane protein composition, size and mechanical properties on single small EVs (sEVs) are simultaneously studied by combined fluorescence and atomic force microscopy. Furthermore, their correlation and heterogeneity in different cellular sources are investigated. The study, performed on sEVs derived from human embryonic kidney 293, cord blood mesenchymal stromal and human acute monocytic leukemia cell lines, identifies both common and cell line-specific sEV subpopulations bearing distinct distributions of the common tetraspanins (CD9, CD63, and CD81) and biophysical properties. Although the tetraspanin abundances of individual sEVs are independent of their sizes, the expression levels of CD9 and CD63 are strongly correlated. A sEV population co-expressing all the three tetraspanins in relatively high abundance, however, having average diameters of $<100 \mathrm{~nm}$ and relatively low Young moduli, is also found in all cell lines. Such a multiparametric approach is expected to provide new insights regarding EV biology and functions, potentially deciphering unsolved questions in this field.

\section{Introduction}

Extracellular vesicles (EVs), capable of transmitting biologically active macromolecules, are emerging as key players in intercellular communication. Thus, they have the potential to be used both as non-invasive diagnostic markers as well as therapeutic agents. ${ }^{[1,2]}$ EVs are a heterogeneous group of lipid-bilayer nanovesicles (30-2000 $\mathrm{nm}$ in diameter) that are secreted by almost all cells and released into the extracellular space. ${ }^{[3]}$ They are broadly divided into three main categories: exosomes (30-150 nm), originating from the endolysosomal pathway, microvesicles (MVs, 50-1000 nm), formed by direct outward budding of the plasma membrane, and apoptotic bodies (500-2000 nm), derived from apoptotic cells. ${ }^{[4]}$ However, in recent years it has been increasingly understood that cells secrete subpopulations of EVs that make them functionally more diverse. ${ }^{[5-8]}$ EVs can impact
S. Cavallaro, F. Stridfeldt, Prof. J. Linnros, Dr. A. Dev

Department of Applied Physics

School of Engineering Sciences

KTH Royal Institute of Technology

Stockholm 10691, Sweden

E-mail: saracav@kth.se; apurbad@kth.se

Dr. F. Pevere, S. S. Sahu, Dr. A. Dev

Department of Electrical Engineering

The Ångström Laboratory

Uppsala University

Uppsala 75121, Sweden

E-mail: apurba.dev@angstrom.uu.se

The ORCID identification number(s) for the author(s) of this article can be found under https://doi.org/10.1002/smll.202008155.

(C) 2021 The Authors. Small published by Wiley-VCH GmbH. This is an open access article under the terms of the Creative Commons Attribution License, which permits use, distribution and reproduction in any medium, provided the original work is properly cited.

DOI: 10.1002/smll.202008155

\author{
Clinical Research Center \\ Department of Laboratory Medicine \\ Karolinska Institutet \\ Stockholm 17177, Sweden \\ Dr. A. Görgens, D. Gupta, Dr. S. El Andaloussi \\ Evox Therapeutics Limited \\ Oxford Science Park \\ Oxford OX4 4HG, UK \\ Dr. A. Görgens \\ Institute for Transfusion Medicine \\ University Hospital Essen \\ University of Duisburg-Essen \\ 45141 Essen, Germany \\ C. Paba \\ Politecnico di Torino \\ Turin 10129, Italy
}

Dr. A. Görgens, D. R. Mamand, D. Gupta, Dr. S. El Andaloussi 
neighboring cells or cells at a distance, as they contain lipids, proteins, or nucleic acid species from the source cell, and have the unique ability to convey these macromolecules via a highly advanced system of intercellular communication. Consequently, they play an important role in numerous physiological and pathophysiological processes, including immune regulation and cancer development. ${ }^{[4,9]}$ Different cell types may produce distinct repertoires of vesicles, reflecting the physiological state of the cell. ${ }^{[10]}$ Understanding the EV biology and physiological relevance, therefore, requires the capacity to resolve their phenotypic variations and identify their functional relations. However, the high heterogeneity of EVs even from a single cell type renders analyses by most available technologies rather ineffective, as they are predominantly based on average properties. ${ }^{[1]]}$ Therefore, there has been an increasing effort for the analysis of EVs in general and small EVs (sEVs)/exosomes in particular at a single particle level. ${ }^{[12-18]}$ On the contrary, the analysis of single sEVs on a sufficiently large and representative population is also extremely challenging, as their sizes lie well below the optical resolution and they are very weak light scatterers. ${ }^{[19]}$ In addition, many $\mathrm{SEV}$ properties, such as the abundance and types of surface proteins, their sizes, genetic cargo, mechanical properties, etc., all seem to play important roles in EV functions, ${ }^{[13,20]}$ indicating the need to include a number of these parameters to identify subtypes within a given SEV population. Most of the reported single vesicle studies so far have only been able to identify SEV subpopulations based on their protein expressions, investigated mainly by immunofluorescent staining approaches. ${ }^{[12,15,18]}$ Although these studies reveal various interesting features of distinct EV subtypes, they may not be sufficient to fully address the issue of heterogeneity. Going a step ahead, recently, Tian et al. successfully combined immunofluorescent staining with side scattering technique to simultaneously measure both the size and protein expression of single EVs, identifying previously unknown EV subtypes. ${ }^{[13]}$ Daaboul et al. also achieved similar results by combining antibody-based capture with interferometric imaging. ${ }^{[14]}$ Recent studies investigating the EV mechanical and structural properties have also found various EV subpopulations bearing distinct mechanical behavior, ${ }^{[21,22]}$ which might be influenced by their parent cells and/or their pathophysiological state, thereby, resulting in variations in their interactions with cells. ${ }^{[21,23]}$ These observations obviously raise new questions, as to which degree are these properties correlated? If not, then what relation do they carry with their parent cells?

Although intriguing, such a study would require a platform that can accurately determine the size, membrane protein composition, and mechanical properties of SEVs, in their physiological environment. However, these properties cannot be simultaneously measured by the available technologies, such as nanoparticle tracking analysis (NTA), interferometric reflectance imaging (IRI), resistive pulse sensing (RPS), etc. Moreover, the size estimation performed by such techniques is limited in accuracy and/or detection range. ${ }^{[2]}$ By combining fluorescence (FL) and atomic force microscopy (AFM), we performed, for the first time, such a multiparametric analysis on single sEVs derived from three different cell lines, namely human embryonic kidney 293 (HEK293), cord blood mesenchymal stromal (cbMSC), and human acute monocytic leukemia (THP1), respectively. In our study, the AFM, installed on top of the fluorescence microscope and aligned with the optical axis, allowed accurate combined measurements on individual sEVs, thanks to a very precise spot identification approach. To minimize size/shape alteration and damage of the soft nanovesicles, force curve-based imaging (or quantitative imaging, QI) mode was performed in a liquid environment. ${ }^{[25]}$ Aiming to delineate the sEV heterogeneity manifested in their molecular, morphological, and mechanical properties, we investigated the abundance of common tetraspanins, for example, CD9, CD63, and CD81 by an immunostaining approach and combined that with high resolution AFM analysis of their sizes and stiffnesses (Young Modulus, YM). The results confirmed the high heterogeneity of the sEVs in their physical and molecular properties, highlighting the presence of SEV subpopulations with possible different functions/biogenesis routes. Overall, our data revealed the presence of vesicles with a large range of diameters $(30-200 \mathrm{~nm})$, Young moduli $(\approx 0.1-25 \mathrm{MPa})$ along with a large variation in the distribution of the investigated membrane proteins. While sEVs from all the cell lines displayed a correlation between the expression levels of CD9 and CD63, the abundance of the investigated tetraspanins were found to be independent of the SEV diameter. In addition, some common and cell-line specific features were also observed in their molecular, morphological, and mechanical properties, which highlight the prospect of the proposed method for highly accurate and multiparametric EV analysis.

\section{Results}

\subsection{Single EV Technology}

Figure 1 schematically demonstrates our measurement platform. sEVs were captured and conjugated to a glass coverslip by using covalent coupling via glutaraldehyde(GA)-amine interaction (Figure 1A, see Experimental Section for details). ${ }^{[26]}$ This was necessary to ensure stability of the SEVs during the entire investigation period, as needed for AFM imaging as well as for immunostaining. Moreover, it assures that the SEV capture protocol is efficient, that is, capable of capturing a significant population from the sample volume and is unbiased to size and expression level. We first tested and optimized the method with HEK293 cell line-derived sEVs, engineered to overexpress CD63 tagged with mNeonGreen (mNG) protein. ${ }^{[27]}$ For these $\mathrm{SEV}$, referred as mNG-EVs hereafter (Figure 1A, top), the sEVconjugation step was followed by PBS washing and combined FL-AFM imaging. For the wild type sEVs (HEK293, cbMSC and THP1) used in the study, referred as wt-EVs hereafter (Figure 1A, bottom), this SEV conjugation step was followed by: i) surface blocking using tris-ethanolamine (Tris-ETHA) and casein, to minimize non-specific binding (NSB) of the antibodies; ii) fluorescently tagged antibody incubation; and iii) washing steps prior to imaging (see Experimental Section for details).

On these wt-EVs, we analyzed the distribution of the widely abundant tetraspanins CD9, CD63, and CD81. ${ }^{[18]}$ It is well-known that the size of the antibodies $\left(\approx 5-10 \mathrm{~nm}^{[28]}\right)$, in addition to various degrees of abundance of their respective membrane proteins, may produce significant steric hindrance. 
A
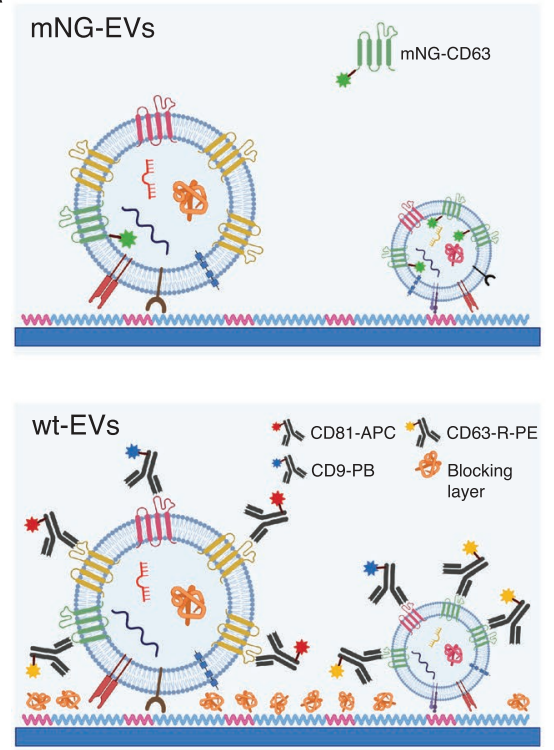

B

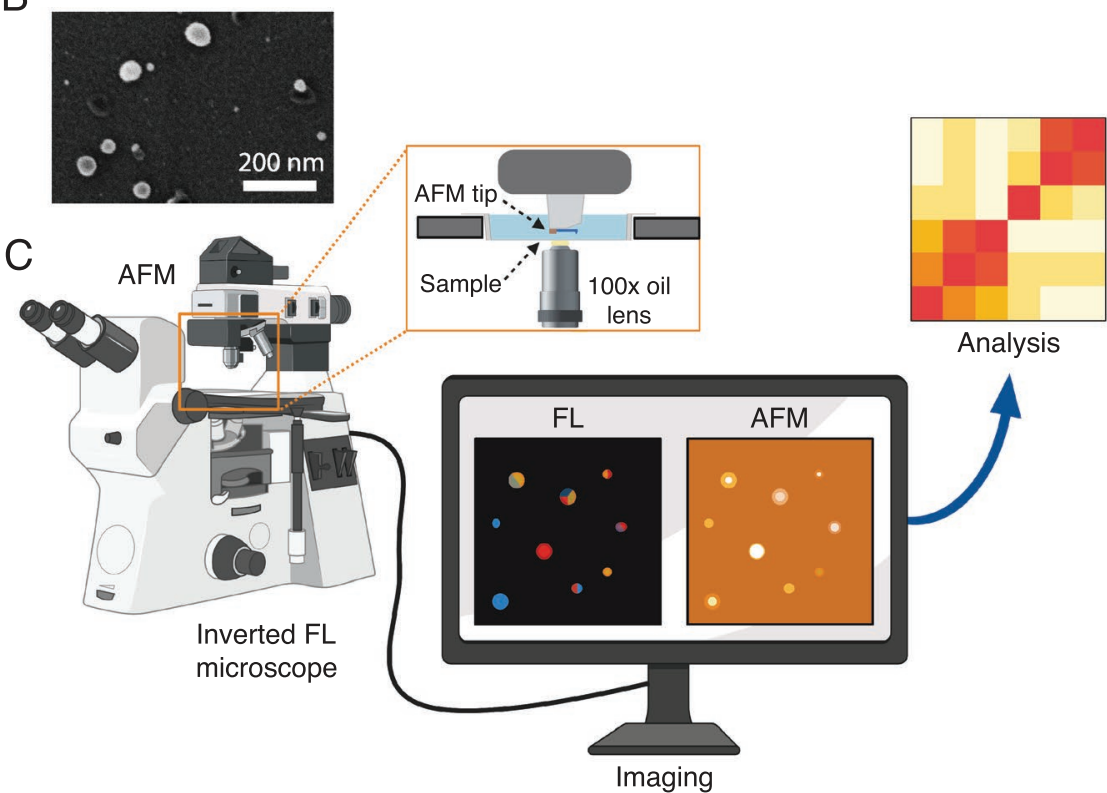

Figure 1. Schematic of the single EV platform. A) Schematic of the sEV capture procedure for both engineered mNeonGreen tagged $s E V s$ ( $m$ NG-EVs) and wild type sEVs (wt-EVs). While the $\mathrm{mNG}$-EVs had the fluorescent $\mathrm{mNG}$-CD63 construct, the wt-EVs were targeted using three different fluorescently labelled antibodies. B) Representative SEM image of the mNG-EVs. C) Schematic of the imaging platform, including inverted FL microscope below the sample support with the EV substrate (inset) and AFM above it, and aligned with optical axis. The FL and AFM images were visualized on a screen and then processed and analyzed using different software. This figure was realized using BioRender.com.

This is particularly true when the antibodies are conjugated in sequence, ${ }^{[29]}$ leading to a notable error in determining the relative membrane protein levels. In order to minimize such an error, we prepared a solution containing all the three fluorescently labelled antibodies recognizing these markers, and let it incubate with the sEVs for $2 \mathrm{~h}$. Particularly, the solution included anti-CD81-Allophycocyanin (CD81-APC, 2 nм) antibody, anti-CD63-R-Phycoerythrin (CD63-R-PE, 2 nм) antibody, and anti-CD9-PacificBlue (CD9-PB, 2 nм) antibody in $1 \times$ PBS (see Experimental Section for details). The selected fluorophores could be imaged simultaneously, showing negligible crosstalk (Figure S1, Supporting Information). Both the mNG-EVs and wt-EVs were isolated using ultrafiltration (UF) and bind-elute size exclusion chromatography (BE-SEC). ${ }^{[30]}$ The purity and yields of sEVs isolated by this method have already been reported in our previous articles, ${ }^{[30,31]}$ however, we further investigated it by using scanning electron microscopy (SEM). The results are presented in Figure $1 \mathrm{~B}$ and Figure S2, Supporting Information. As shown, the data revealed that the sample was mostly composed of spherical objects in the reported size range of sEVs, with a negligible presence of small particles/contaminations of random shape/aggregations. Furthermore, the isolated vesicles were characterized by NTA (Malvern system) and Western Blot (WB, for mNG-EVs)/multiplex bead-based flow cytometry assay (MACSPlex Exosome Kit, for wt-EVs, see Experimental Section for details). Figure S3, Supporting Information, shows the results of such sEV characterization. After the antibody incubation and washing steps, we performed combined FL-AFM imaging, using the setup illustrated in Figure 1C. The FL images were used to qualitatively estimate the abundance of the analyzed tetraspanins on individual sEVs, while the AFM scans were used to estimate their sizes (equivalent diameters) and mechanical properties (Young Moduli). The measurements were performed in liquid (1× PBS) by inserting the substrate in a transparent well (Figure 1C, inset), which was placed in between the objective lens and AFM tip for the combined FL-AFM approach.

\subsection{Platform Characterization on Engineered mNG-EVs}

As mentioned before, we first optimized and characterized the platform with mNG-EVs to ensure that: i) the captured EVs remained stable during the entire investigation period, including washing steps and measurements; ii) the EV capture protocol was efficient and unbiased towards EV size and protein expression; iii) the platform had sufficiently large dynamic ranges for size and surface expression measurements; and iv) the approach discriminated single particles. Figure 2A illustrates a representative FL image of covalently captured mNG-EVs, showing distinct bright spots that could be assigned to single particles. It should be noted that the size of the fluorescence spots in Figure 2A does not correspond to the actual size of the vesicles. In the fluorescence scan, brighter vesicles have larger fluorescence spread. A control measurement performed on an identical substrate but without any sEVs clearly showed no such detectable luminescent spots (Figure S4, Supporting Information). In order to investigate the stability of the captured sEVs and emulate the conditions necessary for antibody staining, we assessed the effect of multiple washing steps on the retention of the sEVs on the substrate using FL imaging. The measurements revealed that 
A

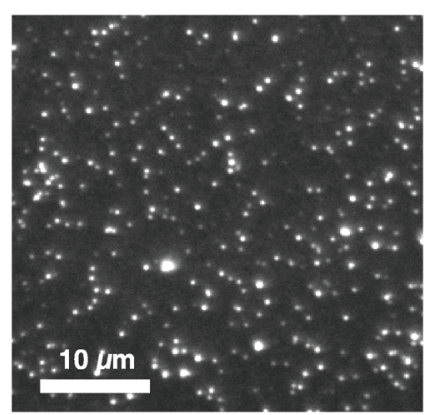

FL scan

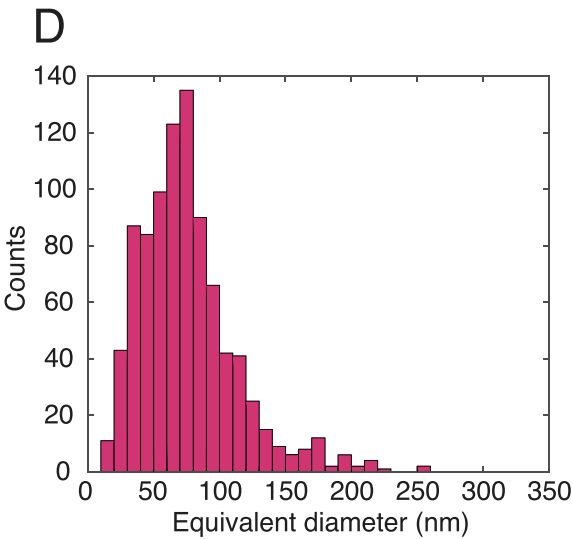

B

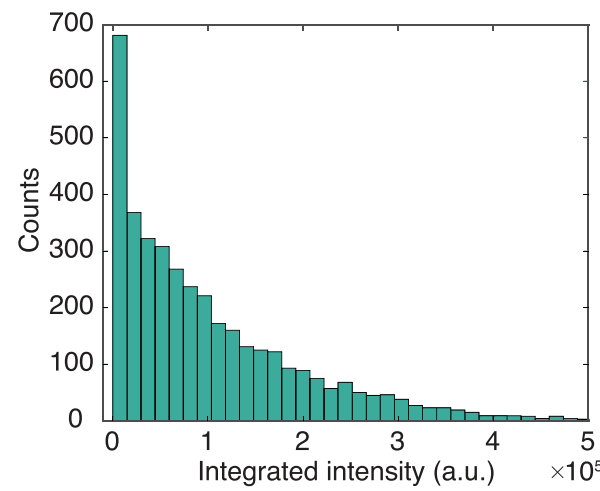

C

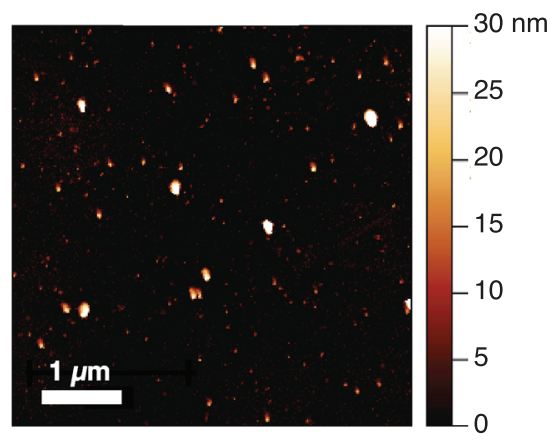

Height measured (AFM)

\section{$\mathrm{F}$}

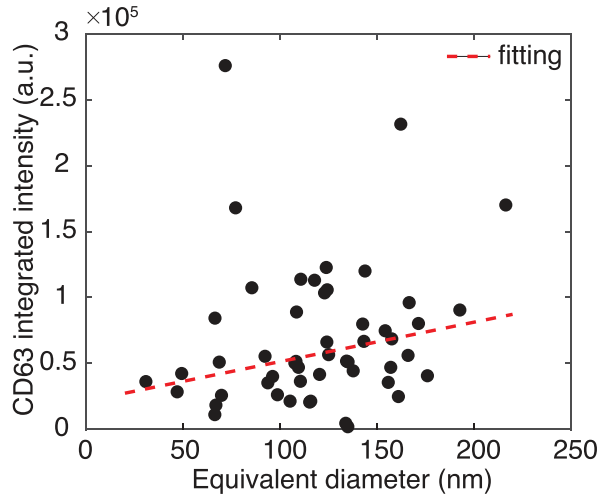

Figure 2. Platform characterization and optimization with mNG-EVs. A) Representative FL image of $m N G$-EVs captured on a glass substrate. B) FL integrated intensity distribution of the captured $\mathrm{mNG}$-EVs. C) A representative AFM scan in liquid of mNG-EVs captured on a separately prepared glass substrate. Height threshold for EV identification was set at $15 \mathrm{~nm}$. D) AFM equivalent diameter distribution of the captured mNG-EVs (height $>$ $15 \mathrm{~nm}$ ), obtained assuming surface area conservation. Distribution obtained from 17 scans of $5 \mu \mathrm{m} \times 5 \mu \mathrm{m}\left(\right.$ total area $=425 \mu \mathrm{m}^{2}$ ) $\mathrm{E}$ ) Representative image of the combined FL (outer image with diffuse encircled spots) and AFM (inner image with yellow solid particles) experiments. F) Correlation plot between the size (equivalent diameter, $x$-axis) and the fluorescence intensity (integrated intensity, $y$-axis) of single vesicles. The fitted curve was obtained by Bi-square linear fitting.

the vesicles remained immobilized even after multiple washings with PBS or water, with a particle variation $<1 \%$ after the third washing step (Figure S5, Supporting Information). Furthermore, the vesicles remained attached to the substrate for at least $12 \mathrm{~h}$, indicating that the covalent capture ensured sufficient EV stability (Figure S5, Supporting Information). To evaluate the capture efficiency of our protocol, we counted the number of fluorescence spots for different sEV concentrations and compared the results with the unitary slope derived from NTA. For the analysis, we first optimized the surface conjugation protocol to ensure that almost all the EVs from the solution interacted and attached to the substrate homogeneously and with minimum losses. Considering homogeneous immobilization, we then converted the area density of particles to the initial volume concentration. As Figure S6, Supporting Information, shows, the number of captured sEVs was 3 times lower than the nominal particle counts obtained by NTA, indicating a capture efficiency of $33 \%$ only. However, given that NTA also detects non-EV particles, ${ }^{[32]}$ that not all the EVs expressed the mNG-CD63 construct and some weakly luminescent EVs may exist below the detection limit, we expected our capture protocol to be far more efficient than $33 \%$ (Figure S6, Supporting Information). For protein expression analysis, we considered the integrated FL intensity distribution (Figure 2B), obtained after background subtraction (Zeiss Data Analysis software, see Experimental Section for details). The data showed an exponentially decreasing FL distribution ranging from $\approx 680$ to $\approx 5 \times 10^{5}$ a.u., with increasing number density towards the low intensity values.

Next, we optimized the AFM parameters by separately measuring sEVs in liquid using soft tips specially designed for biological samples (see Experimental Section for details). Figure 2C and Figure S7, Supporting Information, show representative AFM height images obtained from a substrate with mNG-EVs and an identically prepared control substrate without sEVs, respectively. The scans show clear differences between the substrates with sEVs (Figure 2C) and without (Figure S7, Supporting Information) for a particle height higher than $15 \mathrm{~nm}$, which was set as a threshold for sEV identification. Since the vesicles appeared to assume a flattened shape on the substrate, with the diameters being about twice the heights, for further analysis we considered the sEV equivalent diameters which were estimated by assuming surface area conservation. ${ }^{[33]}$ The equivalent diameter distribution in Figure 2D suggests that $\approx 25 \%$ of the total number of particles had diameters below $50 \mathrm{~nm}$ and that the mean particle diameter was $\approx 76 \mathrm{~nm}$. The 
presence of such small particles could be due to small sizedEVs/exomeres and/or proteins/protein aggregates that often remain undetected by NTA and scattering-based methods. ${ }^{[24]}$ The peak of the distribution appeared at around $70 \mathrm{~nm}$, which is shifted by $40 \mathrm{~nm}$ as compared to the size estimation performed by NTA (Figure S3A, Supporting Information). Overall, both the FL intensity and AFM diameter distributions showed that our capture protocol was unbiased towards the EV size and protein expression. Furthermore, the sufficiently large dynamic ranges of such distributions (including but not limited to $680-5 \times 10^{5}$ a.u. for FL intensity and $10-250 \mathrm{~nm}$ for AFM equivalent diameter) confirmed the feasibility of our platform for accurate sEV size and surface marker expression analyses.

Once validated and optimized separately, we performed combined FL and AFM measurements on the mNG-EVs. Figure $2 \mathrm{E}$ and Figure S8, Supporting Information, show representative images from such measurements of different substrate areas, obtained by overlapping the FL images with the AFM scans of the same spots. Since surface roughness, small spatial shifts in AFM scan, etc. can introduce significant uncertainty in the precision of the image overlap, despite having the optical axis alignment of the two systems, we etched a series of nano- and microscale patterns on the coverslips prior to functionalization. This step was crucial for precise spot identification. Overall, the AFM data could also be used to identify the FL spots corresponding to a single $\mathrm{SEV}$ (Figure 2E, green circles) or groups of multiple particles/sEVs (Figure 2E and Figure S8, Supporting Information, red circles). The latter were excluded from the single particle analysis. As shown, the results suggested that almost all of the fluorescence spots were generated by single EVs, with some exceptions $(\approx 10 \%)$ where a spot corresponded to two or more particles. Figure $2 \mathrm{~F}$ shows the distribution of the mNG-CD63 expression level as a function of the sEV size. For the analysis, we only considered the FL spots corresponding to a single AFM particle and related them to the vesicle equivalent diameters. As suggested from the relatively low slope of the fitting curve and R-square coefficient $(\mathrm{R}$-square $=0.50)$, the number of $\mathrm{mNG}-\mathrm{CD} 63$ proteins was not strongly proportional to the vesicle diameter but was distributed rather stochastically within the expected SEV size range. Moreover, the FL intensity on single vesicles ranged from $\approx 2000$ to $\approx 3 \times 10^{5}$ a.u. (Figure $2 \mathrm{~F}$ ), with most of the sEVs showing an FL count $<1.5 \times 10^{5}$ a.u. This indicated that some of the higher FL intensity values in Figure $2 \mathrm{~B}$ were likely attributed to agglomerated vesicles. Furthermore, the maximum FL intensity was $\approx 100$ times larger than the minimum value. This data range is similar to that reported in other studies, which show that EVs can capture from 1 to $\approx 50-60$ copies of a protein. ${ }^{[13]}$

\subsection{Fluorescence Analysis on wt-EVs}

Once validated, we applied the method to profile wt-EVs from the three different cell lines HEK293, cbMSC, and THP1. In particular, we examined the distribution of the widely abundant tetraspanins CD9, CD63, CD81, and analyzed their dependence on the size and Young Modulus of the corresponding sEVs. The choice of these cell lines was justified by the absence of such a multiparametric study at a single vesicle level and by the possibility to validate our platform with some cell lines that are phenotypically different. ${ }^{[18,27]}$ The selected cell lines are partly studied and have important applications. In this context, HEK293 is the most widely used cell line in EV engineering due to ease of transfection. cbMSC is therapeutically relevant, while THP1 is from myeloid cells. Furthermore, we selected these three tetraspanins because of the lack of understanding of the sEV subpopulations and roles defined by those markers. Figure $3 \mathrm{~A}$ shows a representative FL image of $\mathrm{sEVs}$ stained with all the three antibodies. In the image, the presence of the fluorophores corresponding to CD9, CD63, and CD81 are indicated by the colors blue, yellow, and red, respectively. Distinct bright spots with sufficiently low background clearly indicated good specificity of the immunostaining protocol.

The same was further verified with control measurements, where the antibodies were incubated on functionalized substrates without sEVs (Figure S9, Supporting Information) or where isotype control antibodies were incubated on substrates with sEVs (Figure S9, Supporting Information). The FL results presented in Figure 3 also show the differences among the analyzed samples, in terms of both the abundances and distributions of the tetraspanins within the sEV populations. In particular, Figure 3B shows the number of sEVs that were detected to express the different tetraspanins in each of the cell lines, from a microscopic area $(133.12 \mu \mathrm{m} \times 133.12 \mu \mathrm{m})$. Since the original concentrations of the isolated particles were different among the cell lines (NTA analysis in Figure S3, Supporting Information), the samples were appropriately diluted from their initial stocks in order to make identical concentrations for all cell lines, prior to their immobilization to the substrate. Thereafter, by taking an identical volume for all cell lines, we ensured that the total number of particles considered from each sample remained similar. Furthermore, as the sEV capture protocol was unbiased to the membrane proteins (vesicles not captured via surface protein but via covalent amine-coupling) and was carried out for the same time interval, we expected a similar number of vesicles binding to the substrate for all the cell lines. Therefore, Figure 3B could be used to compare the populations of sEVs that were positive to the different tetraspanins in these cell lines. The results (Figure 3B) revealed that for the HEK293 cell line, the number of sEVs positive to each of the tetraspanins were similar, with a slight enrichment in CD81 expressing sEVs. The cbMSC sEVs, instead, were more abundant in CD63 positive sEVs than the other two. ${ }^{[34]}$ Similar to HEK293, the THP1 sEVs also did not show prevalence of a particular marker (Figure $3 \mathrm{~B}$ ). Figure $3 \mathrm{C}$ presents a box plot graphically depicting the distribution of the three tetraspanins on single vesicles. Here, for each tetraspanin we considered its total integrated intensity on single vesicles and then plotted the distribution for all the SEVs positive for that marker. This was repeated for all cell lines separately. Being such integrated intensities proportional to the number of tetraspanins on each vesicle, the data showed that the HEK293 sEVs had the highest abundance of CD81 per sEV compared to the other two cell lines. The THP1 sEVs showed instead the highest levels of CD63 per sEV, while none of the analyzed cell lines showed prevalence of CD9 per vesicle. When analyzing the $\mathrm{SEV}$ population expressing the three tetraspanins simultaneously, the results revealed interesting correlations, as presented in Figure 3D. In particular, 
A

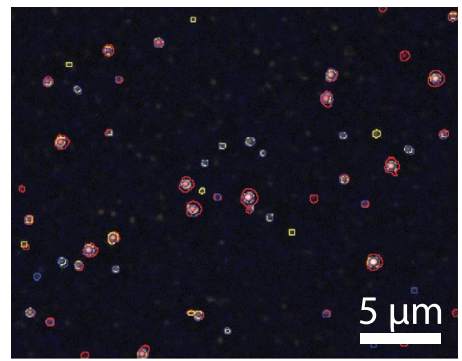

FL scan
B

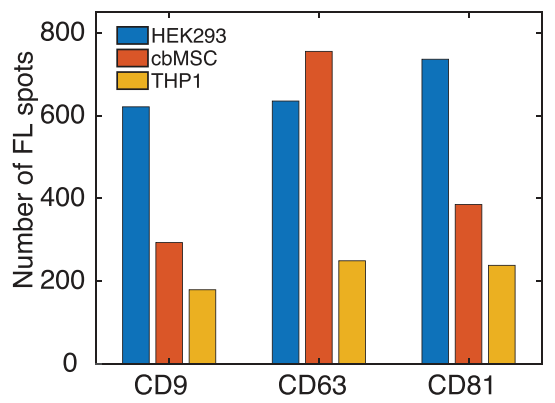

cbMSC EVs

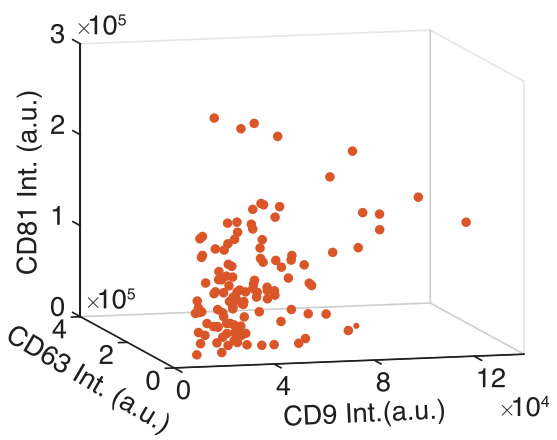

C

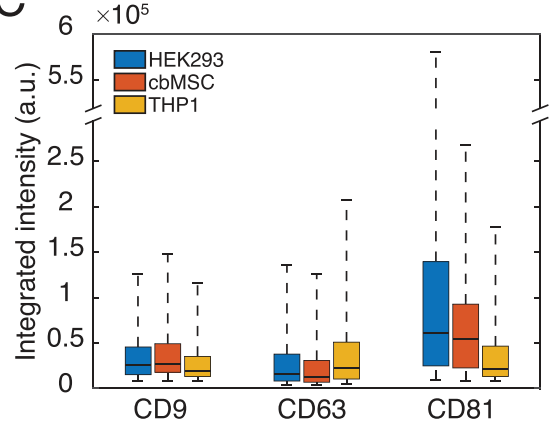

D

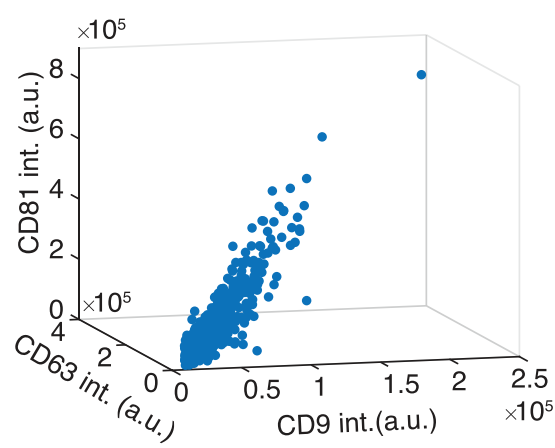

$E$

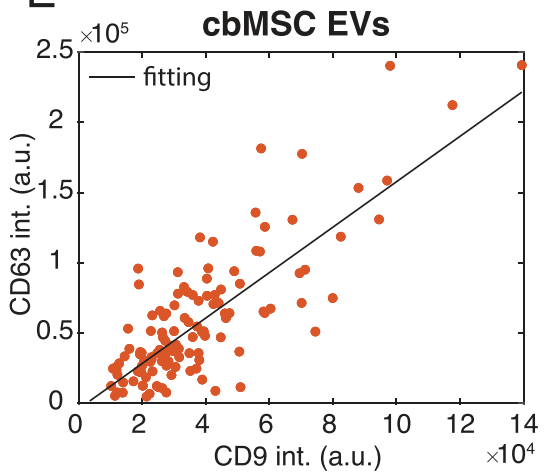

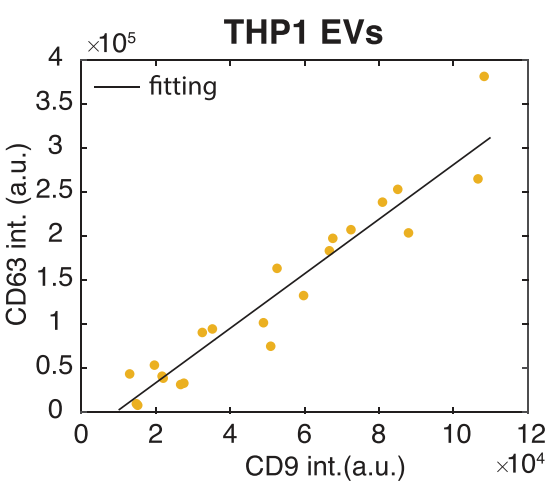

$F$
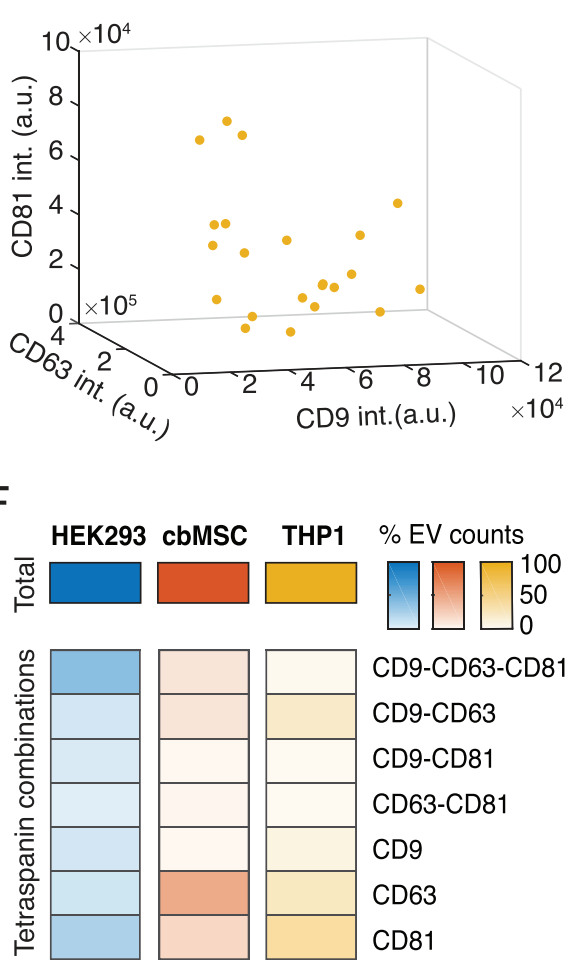

Figure 3. FL analysis on the three wt-EV samples HEK293, cbMSC, and THP1. A) Representative FL image of EVs targeted with CD9-PB, CD63-R-PE, and CD81-APC antibodies, showing vesicles co-expressing all markers simultaneously (three colors) and vesicles only expressing two or one markers. B) Number of positive sEVs detected in a microscope area for each tetraspanin analyzed and cell line. C) Box plots summarizing the integrated intensity distributions for all the markers and samples analyzed. Whisker value set to 4. D) Correlation of the FL integrated intensities for the CD9-CD63-CD81 positive EVs for the HEK293 (left, blue), cbMSC (middle, red), and THP1 (right, yellow) EVs, respectively. E) Correlation of the CD9 and CD63 FL intensities for the CD9-CD63-CD81 positive EVs of cbMSC (red) and THP1 cell lines (yellow). F) Heatmap showing the amount of sEVs present for each protein combination subgroup. For example, the CD9-CD63-CD81 label indicates the number of EVs positive for the three markers simultaneously, CD9-CD63 the number of EVs positive only for CD9 and CD63, whereas CD9 the number of EVs only positive for CD9 protein (no CD63, no CD81 detected). Numbers of EVs per subgroup expressed as a percentage of the total number of EVs detected for each cell line (100\%).

we observed that, while for the HEK293 sEVs, the increase of one marker, for example, CD9, was accompanied by a linear increase of the other two markers, that is, CD63 and CD81 $\left(\mathrm{R}\right.$-square $\mathrm{tot}=0.84, \mathrm{R}-\mathrm{square}_{\mathrm{CD9}-\mathrm{CD} 63}=0.88, \mathrm{R}-\mathrm{square}_{\mathrm{CD} 9-\mathrm{CD} 81}=$ 0.83 , R-square $\mathrm{CD}_{\mathrm{C} 63-\mathrm{CD} 81}=0.81$ ), for the cbMSC and THP1 EVs, there was not a similar correlation among all the three tetraspanins ( $R$-square tot $=0.2$ and R-square tot $=0.27$, respectively). However, the intensities of CD9 and CD63 were correlated for the cbMSC and THP1 sEVs in this group, with both linearly increasing with each other (Figure 3E, R-square $=0.72$ for cbMSC and R-square $=0.92$ for THP1 EVs). The same correlation between these two tetraspanins was also observed for the sEV populations only positive to CD9-CD63 for all the three cell lines (Figure S10, Supporting Information), indicating that this is a general feature of the cell lines studied here. The data also showed remarkable differences in the distribution of the marker combinations among the samples. This is highlighted in the heatmap in Figure 3F, which illustrates the proportions 


\section{A HEK293 sEVs}

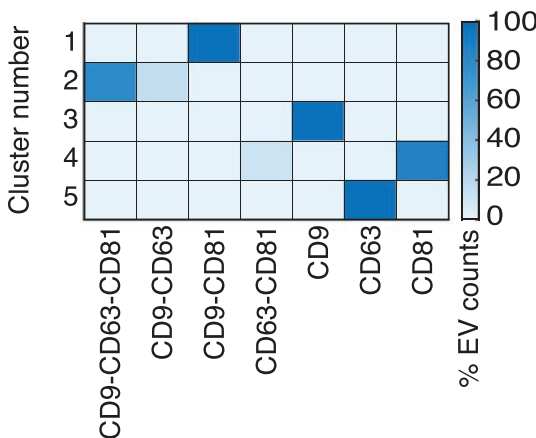

B

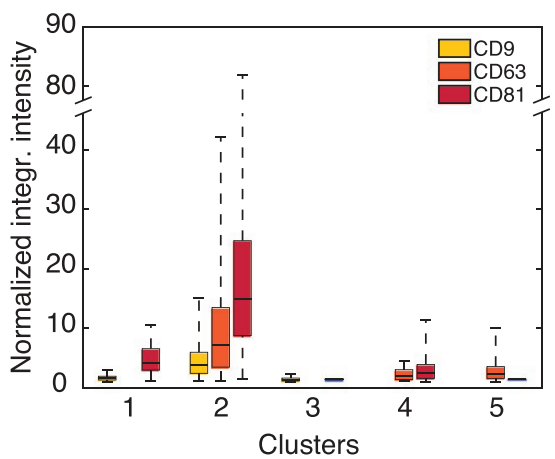

cbMSC sEVs
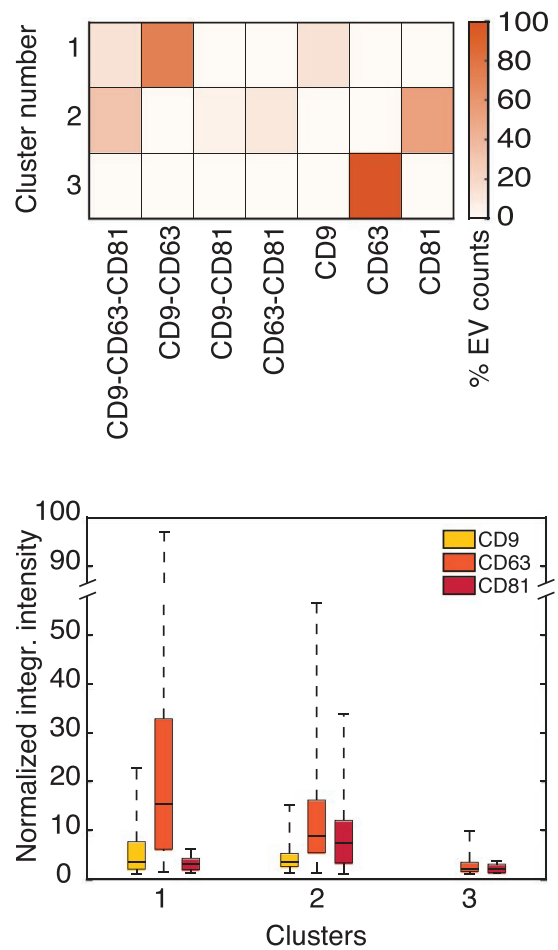

\section{THP1 sEVs}
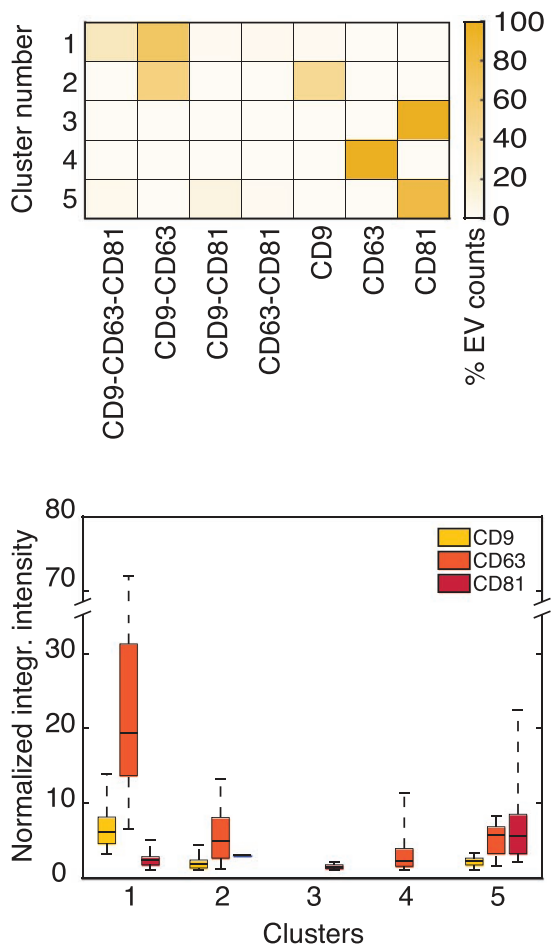

Figure 4. Clustering analysis on the HEK293, cbMSC, and THP1 wt-EVs. The results from the different cell lines are separated in three different columns. A) Heatmaps of the tSNE derived marker expression profiles, showing the tetraspanin combinations within the identified clusters (5 for HEK293 EVs, 3 for cbMSC EVs, and 5 for THPI EVs). B) Box plots of the normalized FL intensity distributions of the three tetraspanins for the tSNE derived clusters.

of different EV subpopulations categorized according to their tetraspanin combinations. As presented, both the HEK293 and cbMSC cell lines showed a similar amount of total tetraspanin positive sEVs (992 and 1005, respectively) in the investigated microscopic area, while for THP1, the amount stood at nearly $50 \%$ (502 tetraspanin positive sEVs detected). Within their respective populations, the fraction that contained all the three tetraspanins were about $40 \%, 12 \%$, and $4 \%$, respectively for HEK293, cbMSC, and THP1 EVs. This indicated that the co-presence of all the three tetraspanins was a far more dominant feature of the HEK293 sEVs than the other two. On the other hand, the cbMSC sEVs seemed to dominantly favor vesicles only positive for CD63 ( $\approx 48 \%$ of the population), while the THP1 sEVs showed a preference to vesicles only positive for CD81 ( $\approx 38 \%$ of the population). Another common feature, as appears under the group of sEVs co-expressing only two tetraspanins simultaneously (Figure $3 \mathrm{~F}$ ), is that the co-enrichment of CD9-CD63 combination was favored compared to the other two tetraspanins combinations among all the three cell lines. This was particularly dominant in THP1 EVs, where $\approx 20 \%$ of the total population displayed this feature.

\subsection{FL Clustering Analysis on wt-EVs}

In order to understand how the abundance of the various tetraspanins per sEV influences the subpopulations identified in Figure 3F, we mapped the sEV FL intensity data onto a 2D plane using t-distributed stochastic neighbor embedding (tSNE) method. For the analysis, we first optimized the mappings for each cell line according to perplexity values and convergence (see Experimental Section for details). The results of the analysis are presented in Figure S11, Supporting Information, where each point represents a single vesicle. We emphasize here that the abundance of the tetraspanins was qualitatively compared by taking the FL intensity of each tetraspanin channel normalized by the minimum detectable intensity (weakest spot from a single sEV) for the same channel and cell line. This value is proportional to the amount of each type of protein present on each vesicle. As shown in Figure S11, Supporting Information, the optimal parameters identified five main clusters for the HEK293 sEVs, three clusters for cbMSC sEVs, and five clusters for the THP1 sEVs. Figure 4A presents the constituent tetraspanin combinations in each of the identified clusters, while Figure 4B shows the distributions of their FL intensity values. The analysis suggested that for the HEK293 sEVs, the most populated cluster (cluster 2, 49\% of the sEV population, Figure 4A and Figure S11, Supporting Information) was composed mainly by the vesicles expressing the three tetraspanins simultaneously and by those only positive for CD9-CD63 (Figure 4A). Although the intensities spread over a large range (cluster 2, Figure 4B), on average this group of vesicles showed the highest intensity values for all the tetraspanins, indicating an enrichment of such markers. The second most populated cluster (cluster 4, 26\% of the sEV population, Figure 4A and Figure S11, Supporting Information), in this cell 
A

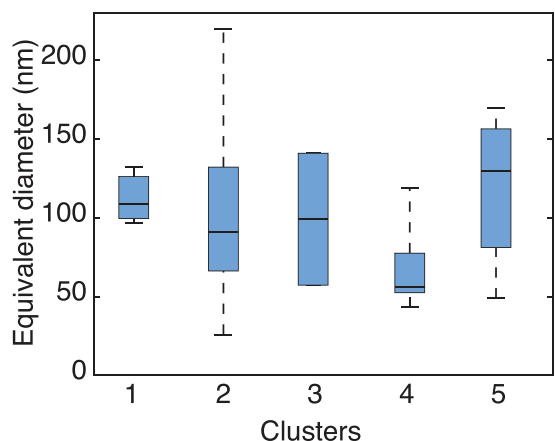

B

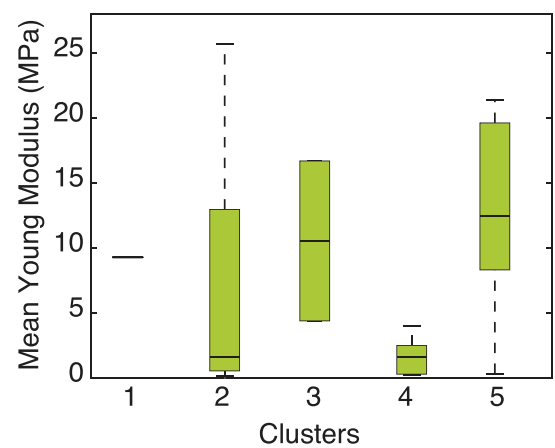

cbMSC sEVs
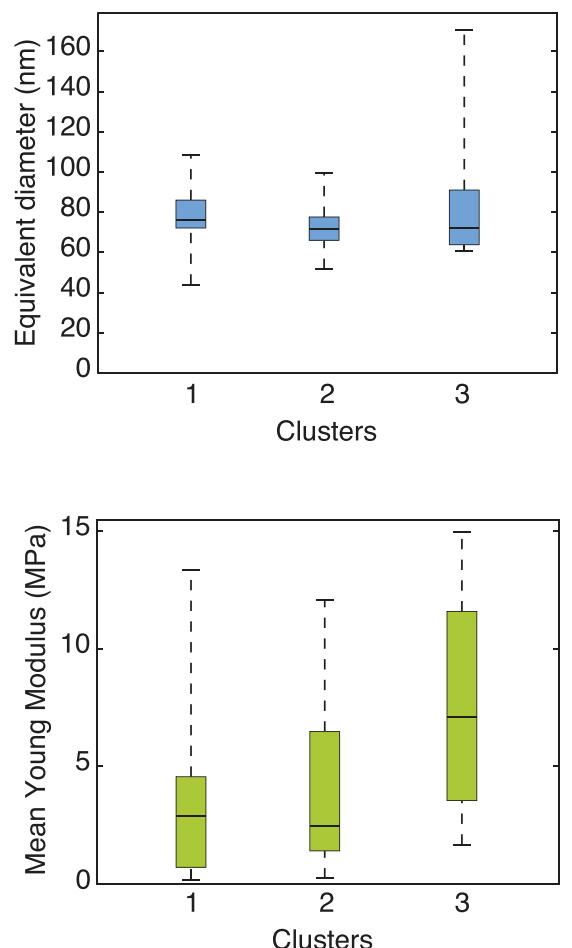

THP1 sEVs
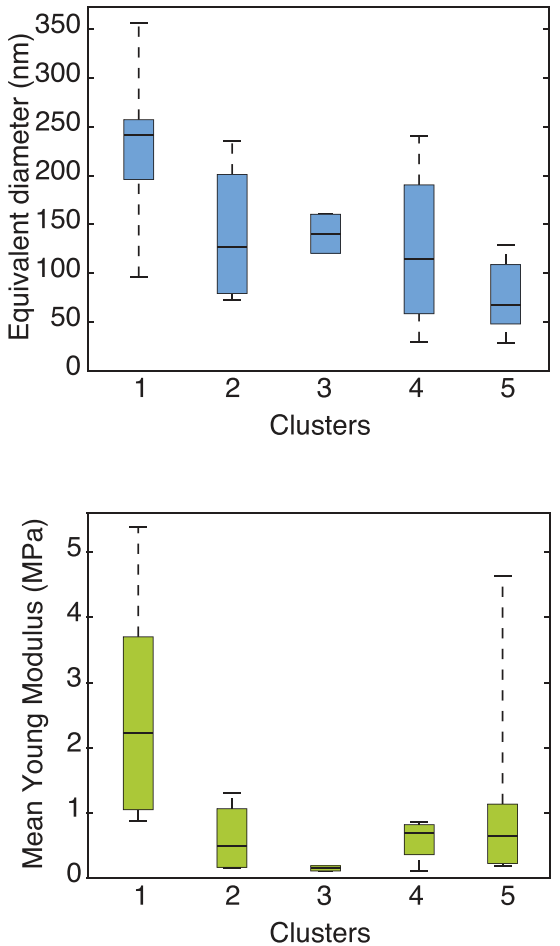

Figure 5. Distribution of A) size and B) Young Modulus of the SEVs analyzed by AFM within the previously identified clusters based on FL intensities.

line was composed of vesicles either positive for CD81 only or for CD63-CD81. Intensity comparison showed that compared to cluster 2 (Figure 4B), all the other clusters showed low levels of tetraspanin abundance per vesicle.

The results obtained for the cbMSC sEVs revealed a quite different behavior. In this case, the clustering algorithm identified three main clusters. Unlike HEK293, in this cell line the sEV subpopulation co-expressing all the three tetraspanins split into two clusters, one of them including also the sEVs positive only for CD9-CD63 (cluster 1, 17\% of the SEV population, Figure 4A) and the other one including the sEVs expressing CD81 (cluster 2, 35\% of the sEV population, Figure 4A). Interestingly, the CD9-CD63-CD81 positive vesicles clustering with the CD9-CD63 expressing sEVs (cluster 1) showed the highest levels of CD9 and CD63, while the sEVs clustering with the CD81 expressing vesicles (cluster 2) showed the highest levels of CD81, but intermediate levels of CD9 and CD63. Finally, the data for the THP1 sEVs suggested a similar behavior as the cbMSC vesicles.

\subsection{Distribution of EV Physical Parameters Within Clusters}

Following fluorescence analysis and clustering, we analyzed how the vesicle physical properties, for example, size and YM, related to their protein expressions, in order to further characterize the SEV heterogeneity and investigate the role played by these parameters. As reported earlier, other than size profiling, AFM can also accurately determine particle adhesion, stiffness, or deformability, which may be responsible for different cellular interactions. ${ }^{[21]}$ Figure 5 summarizes these results, showing the distribution of the SEV size (Figure 5A) and YM (Figure 5B) within the identified clusters. The YM values were calculated by averaging over all the pixels on individual vesicles and is being used here a measure of the vesicle stiffness. For this purpose, we considered the vesicles randomly scanned by AFM on different substrate areas of the initially acquired FL area, in order to obtain unbiased measurements (see Experimental Section for details). Figures S12 and S13, Supporting Information, show a representative combined FL-AFM measurement performed on the HEK293 wt-EVs and the AFM size distributions of the analyzed vesicles, respectively. Due to the low throughput of AFM in image acquisition, we could only analyze a fraction of those vesicles identified by fluorescence imaging. This was necessary to ensure the best image resolution. However, the acquired data points were representative of the whole sEV populations (Figures S14 and S15, Supporting Information). As presented in the Supporting Information, the vesicles analyzed by AFM were uniformly distributed among all the different clusters (Figure S14, Supporting Information) and showed similar tetraspanin distributions (Figure S15, Supporting Information) as the whole sEV dataset (Figure 4B).

The size and the YM analysis of the vesicles revealed several interesting features. In particular, for the HEK293 sEVs, the cluster containing the vesicles expressing the three proteins simultaneously (cluster 2) had on average small diameters $(<100 \mathrm{~nm})$, but also the largest variations. The diameters of the $\mathrm{sEVs}$ in this cluster were distributed in the range from $\approx 30$ to 
$\approx 220 \mathrm{~nm}$. On the other hand, the sEVs positive for CD81 only or those expressing CD81 in combination with CD63 (cluster 4) had the smallest mean diameters $(\approx 60 \mathrm{~nm})$. The remaining clusters $(1,3,5)$, containing the sEVs expressing low levels of tetraspanins, showed on average larger diameters. The analysis performed on cbMSC and THP1 sEVs revealed some analogies with the HEK293 cell line but also several differences. In both these samples the sEVs expressing the three tetraspanins, which split between two clusters, showed two different behaviors. In particular, those vesicles coupling with the CD9-CD63 expressing sEVs (clusters 1 for both cbMSC and THP1 sEVs) had on average larger diameters (Figure 5A) than those coupling with the CD81 expressing ones (Figure 5A, cluster 2 for cbMSC sEVs and cluster 5 for THP1 sEVs). For cbMSC, while most of the analyzed sEVs had diameters $<100 \mathrm{~nm}$, the population expressing CD63 only (cluster 3, Figure 5A) showed a large spread in diameter reaching up to $160 \mathrm{~nm}$. The YM values of the sEVs also displayed a very large heterogeneity, as presented in Figure 5B. Although they varied from $170 \mathrm{kPa}$ up to $25 \mathrm{MPa}$, the majority of the sEVs had their average YM below $5 \mathrm{MPa}$, irrespective of the cell line. The mean value of YM in some clusters was different by more than an order of magnitude (e.g., clusters 2 and 5 for HEK293 sEVs, Figure 5B). The clusters displaying relatively small mean YM values for HEK293 and cbMSC cell lines (cluster 4 and 2, respectively), seemed to be abundant with CD81 only positive sEVs (see Figure 4A).

\subsection{Correlation between Tetraspanin Levels and the Physical Parameters}

The tetraspanin's profile of individual vesicles from the three cell lines (presented in Figures 3 and 4) and their corresponding physical parameters (size and YM, as presented in Figure 5) also offered us a unique opportunity to examine if any linear correlation existed among these properties and if so, how strong their statistical significance was. First, we examined the presence of any linear correlation between the abundance of any of the tested tetraspanins and the size (equivalent diameter) of the corresponding vesicle. This is shown in Figure 6A and Figure S16, Supporting Information, where we plotted the expression levels of each of the tetraspanins as a function of the vesicle diameters. Figure 6B and Figure S17, Supporting Information, show the same but as a function of the YM values of the sEVs. Clearly, as seen in Figure 6A, the tetraspanin abundance (intensity) did not increase with the sEV diameter, suggesting that high expressing vesicles did not necessarily have large diameters. The Pearson correlation coefficient for the case of CD9, CD63, and CD81 on HEK293 sEVs were found to be $-0.2,-0.3$, and 0.1 , respectively, indicating a strong statistical significance of this observation. Similarly, the analyses of tetraspanin abundance as a function of YM of sEVs (Figure 6B) and of YM as a function of sEV diameter (Figure S18, Supporting Information) also did not show any linear correlation. The analysis performed on cbMSC and THP1 sEVs, presented in Figures S16, S17, and S18, Supporting Information, revealed analogies with the HEK293 cell line. Interestingly, Figure 6A also shows the trend that the sEVs with high tetraspanin abundance, irrespective of the type, are mostly populated around
$100 \mathrm{~nm}$ in diameter. Since for all the analyzed cell lines, the sEV subpopulation displaying high levels of tetraspanins were those that simultaneously co-expressed all the three tetraspanins, we also analyzed the diameter and YM distributions of this particular sEV subtype. Figure $6 \mathrm{C}$ presents the results of the investigation. For HEK293 and cbMSC cell lines, the majority of the sEVs in this category had diameters below $100 \mathrm{~nm}$ (Figure 6C, left) and relatively low YM (<7-8 MPa, Figure $6 \mathrm{C}$ right). Also THP1 sEVs in this category showed low YM values, however having much larger diameters as compared to the other two cell lines (Figure 6C, left).

\section{Discussion}

Over the past decade, many scientific investigations have highlighted the importance of EVs in intercellular communication and also their role in pathological conditions. ${ }^{[1,2,4,9]}$ EVs, being generated from either cell membrane budding or endosomal invagination, are known to capture cell specific proteins, RNAs or lipids. ${ }^{[6]}$ However, the mechanisms of this cargo packaging remain largely elusive so is the identity of functionally distinct EV subsets. This is because the vesicles carry a large number of molecular and biophysical signatures, which necessitates their individual characterization with an accurate and multiparametric approach to be able to delineate their common and distinct features. Once the subsets are identified, their biological activities and functions can be followed with in vitro immunomodulation assays and/or in animal biodistribution models, thereby revealing the functional role of such an EV subset. In this context the proposed method offers a unique advantage as a number of EV parameters such as their membrane protein composition and abundance, their size and mechanical properties can be investigated with very high resolution and accuracy. As a proof of concept, we developed a sample preparation protocol with $\mathrm{mNG}$-EVs to ensure efficient and unbiased EV capture irrespective of their size (Figure 2D) and protein expression (Figure 2B). The strategy also showed to maintain the stability of the captured vesicles for the investigation. As presented in Figure 2D, and Figures S12 and S13, Supporting Information, AFM can detect particles as small as 10-20 nm, which are below the reported size range of EVs. ${ }^{[4]}$ Although it is still not known whether these particles are EVs or exomeres, ${ }^{[35]}$ the data highlight the capability of the method to characterize EVs in their small size range (i.e., 30-40 nm, Figure S13, Supporting Information) as well as to investigate the purity of the EV isolation methods. However, a major benefit stems from the fact that the combined FL-AFM measurements can also easily discriminate single vesicles from other closely spaced particles or EV groups (Figure 2E) which are difficult to identify by fluorescence and/or scattering based approaches.

The results obtained by analyzing the sEVs from the three different cell lines in general show large heterogeneity in the sEV populations, displaying both common and distinct features among the cell lines. As presented in Figure 3B,C, we found that CD81 is a more frequently and abundantly expressed tetraspanin in HEK293 sEVs, a feature also reported earlier. ${ }^{[36]}$ In comparison, cbMSC sEVs show a prevalence of CD63 among the tested proteins. This is also in agreement with a previous 
A

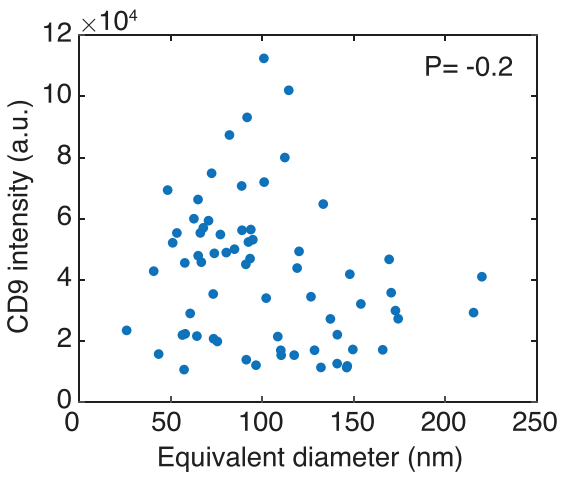

$\mathrm{B}$

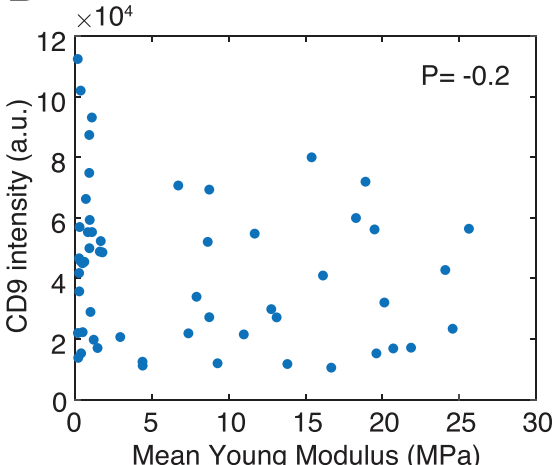

C

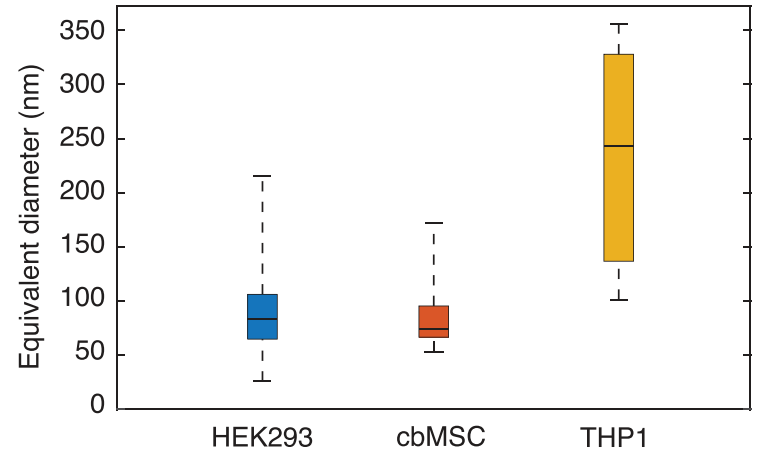

HEK293 sEVs
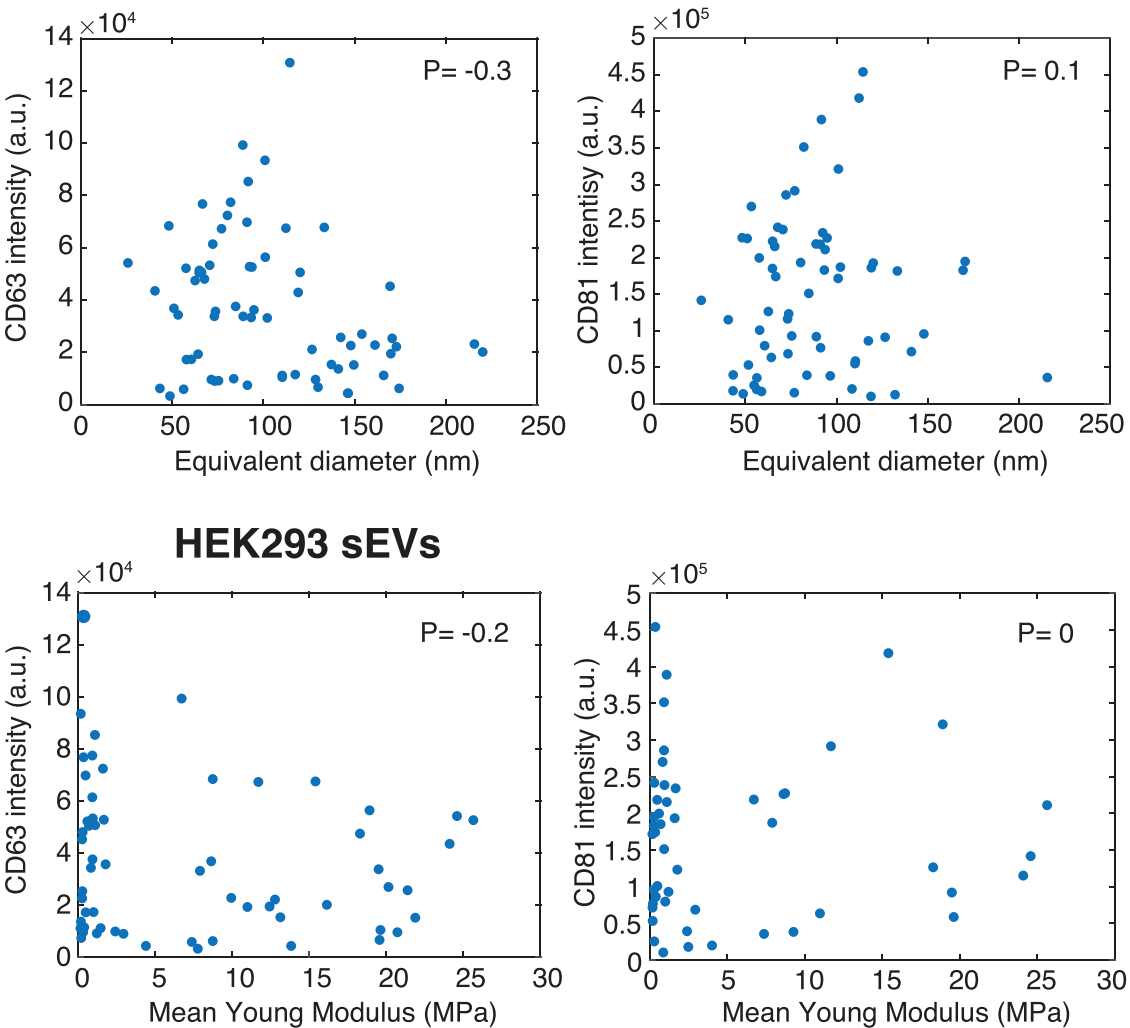

CD9-CD63-CD81 sEVs

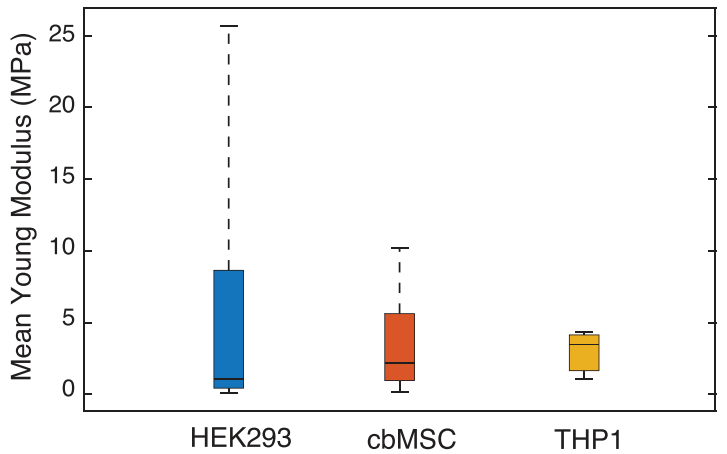

Figure 6. Correlation plots between A) the tetraspanin FL intensities and the sEV equivalent diameters and B) the tetraspanin FL intensities and the sEV YM for HEK293 sEVs. The value of P represents the Pearson coefficient. C) Box plot of the size distributions of the population of sEVs expressing the three tetraspanins simultaneously for all three cell lines.

report. ${ }^{[34]}$ Furthermore, HEK293 sEVs are more enriched with the analyzed tetraspanins compared to the other two samples (Figure 3B), ${ }^{[27]}$ while THP1 sEVs show the lowest numbers of tetraspanin positive vesicles. However, in comparison with the previous reports our combined method has the advantage to complement the protein abundance data with the vesicle size and stiffness (YM), thereby further deciphering EV heterogeneity. For all the cell lines included here, the results also suggested the presence of a co-localization and correlation between CD9 and CD63 proteins, both increasing (or decreasing) with each other on single vesicles. Moreover, for all the cell lines, we detected a sEV subpopulation which was composed of vesicles expressing all the tetraspanins simultaneously and with relatively high expression levels. Since they clustered together with the vesicles only expressing CD9-CD63, the results suggested a possible different biological function of this subgroup of vesicles and/or a different biogenesis route, for example, from plasma or endosome membrane. This behavior has also been hypothesized by other studies on EV markers. ${ }^{[37-39]}$

Moreover, the results also seem to reveal that the analyzed tetraspanins do not follow random distributions but might be encapsulated into the vesicles according to different 
mechanisms. This behavior can be deduced from Figure 3F. A random distribution would result in the population of sEVs expressing just one of the markers to be the dominant one. This would be followed by those sEVs carrying two of the markers and lastly, by the population containing all three markers simultaneously. However, the data do not follow this trend for the HEK293 sEVs. In particular, $43 \%$ of the EV population in HEK293 cell line showed one marker only. This was followed by the EV population expressing three markers simultaneously (40\%) and EVs expressing only two markers (17\%). For the cbMSC and THP1 sEVs instead, most of EVs (>70\%) expressed one marker only and were followed by EVs expressing two $(\approx 20 \%)$ and three markers $(<10 \%)$. The same conclusion can be derived from the results of the combined FL-AFM measurements. A random tetraspanin distribution would also be likely accompanied by an increase in protein expression with sEV size, because of the larger surface area. Although the study by Tian et al. suggested this trend on EVs derived from colorectal cancer cells, ${ }^{[13]}$ our results showed no linear correlation between the FL intensity and the vesicle equivalent diameters. This behavior has also been observed in other investigations, ${ }^{[38]}$ indicating the possibility for selective EV cargo loading and/or different routes of biogenesis. ${ }^{[38]}$ Furthermore, a different correlation between the size and protein expression level may also be the result of differences in the EV isolation procedures and/or cellular source.

The AFM data of the vesicle sizes, combined with the FL data, also reveal that although not all the detected particles below $50 \mathrm{~nm}$ are EVs (these sizes are also detected in control substrates), there exist a population in this size range (Figure S13, Supporting Information) that can be either very small EVs or exomeres. ${ }^{[35]}$ The comparison of the AFM results on the vesicle size and YM also suggested the presence of some common and distinct features among the samples. For example, the subpopulation of HEK293 and cbMSC derived SEVs that contains all the three tetraspanins simultaneously are mostly distributed below $100 \mathrm{~nm}$ in diameter (Figure 6C, left), while the THP1 derived vesicles expressing all the three tetraspanins displayed relatively large diameters. The THP1 cell line also had a lower abundance of vesicle expressing tetraspanins (compared to the other cell lines) and proportions of vesicles co-expressing the three tetraspanins simultaneously. Finally, the YM values of the sEVs were also found to be highly heterogeneous, showing no linear correlation with size or the tetraspanin abundance, irrespective of the cell type. Clearly, the proposed platform goes beyond the state-of-the art in single EV characterization offering a unique opportunity to combine multiple EV parameters with better resolution and accuracy. Application of the technique in other cell line and/or pathological conditions may provide a useful insight about EV heterogeneity and their functional role.

\section{Conclusion}

In conclusion, we present a platform for the high resolution and multiparametric analysis of single sEVs to resolve their heterogeneity. The platform combines AFM and FL microscopy to characterize individual sEVs and profile them based on their membrane protein composition, size, and Young Modulus. The method applied to sEVs collected from the three different cell lines HEK293, cbMSC, and THP1, shows the existence of several subpopulations bearing distinct properties, essentially highlighting the high EV heterogeneity. The data shows both common and cell-line specific features in the SEV populations. Although the sEVs investigated here are distributed in a large diameter range of $30-200 \mathrm{~nm}$, the results do not show any size dependence of the expression levels of the common tetraspanins (CD9, CD63, CD81). However, some of the cell lines show a subpopulation of sEVs that simultaneously expresses all the tested tetraspanins in a relatively high abundance but existing mostly in a narrow size range of 70-100 nm. The level of CD9 and CD63 expressions in SEVs was also found to be strongly related to each other, irrespective of their cellular origin. On the contrary, the relative population of sEVs containing different combinations of the tested tetraspanins was found to depend on the cell line they were derived from. Although the biological significance of these observations is yet to be determined, the combined method shows a significant step forward for the analysis of single vesicles. This combination of properties may provide new insights when analyzing EVs from clinical samples, potentially allowing us to find new/ more accurate diagnostic and/or therapeutic agents as well as help in deciphering unsolved questions regarding EV biology.

\section{Experimental Section}

Reagents: High purity deionized water (DIW) with a resistivity of $18 \mathrm{M} \Omega \cdot \mathrm{cm}$ was used throughout all the experiments. Phosphate-buffered saline (PBS, P4417) in tablets was purchased from Sigma-Aldrich. Anti-CD81-APC (A87789) was purchased from Beckman Coulter; antiCD63-R-PE (1P-343-T100) and anti-CD9-PacificBlue (PB-208-T100) were purchased from ExBio. If not stated otherwise, all of the other chemicals were purchased from Sigma-Aldrich.

EV Purification and Isolation: sEVs were prepared from THP-1 human monocytic cells, HEK293 Freestyle suspension cells (ThermoFisher), and immortalized human cord blood-derived mesenchymal stromal cells (cbMSCs; ATCC PCS-500-010) through ultrafiltration and bind-elute size exclusion chromatography (BE-SEC) as described previously. ${ }^{[40]}$ In brief, conditioned media were pre-cleared by low-speed centrifugation ( $5 \mathrm{~min}$ at $700 \times \mathrm{g}$, then $10 \mathrm{~min}$ at $2000 \times \mathrm{g}$ ) and by filtration through $0.22 \mu \mathrm{m}$ filters (Corning, cellulose acetate, low protein binding) before they were concentrated and diafiltrated with two times the initial volume of PBS by tangential flow filtration $\left(300 \mathrm{kDa}\right.$ MidiKros columns, $370 \mathrm{~cm}^{2}$ surface area, Spectrum Labs). sEVs were further concentrated by using Amicon Ultra-15 10 kDa molecular weight cut-off spin filters (Millipore). Particle concentrations were assessed by NTA with a NanoSight NS500 instrument, as previously described. ${ }^{[27]}$ Fluorescently labelled sEVs were prepared accordingly from HEK293 Freestyle cells which were lentivirally engineered to stably express CD63-mNeonGreen fusion proteins as described before. ${ }^{[27]}$

EV Bulk Surface Characterization: The general surface marker composition for the sEVs from mNG-CD63 HEK293 cells was assessed by Western Blot (WB). The results showed the presence of common exosomal markers, for example, CD9, CD63, and CD81, and the absence of other markers, for example, a GM130 marker typical for apoptotic bodies (data not shown). The general surface marker composition for the sEVs from the three cell sources (HEK293, cbMSC, THP1) was assessed by a multiplex bead-based flow cytometry assay (MACSPlex Exosome Kit, human, Miltenyi Biotec) by using an optimized workflow as described previously. ${ }^{[27]}$ In brief, $1 \times 10^{9}$ NTA-based particles from each sample were incubated overnight in 96-well filter plates (supplied with the kit) with MACSPlex Exosome Capture Beads ( $5 \mu \mathrm{L}$ ) on an orbital shaker at 450 RPM at room temperature. Beads were washed 
with MACSPlex buffer $(200 \mu \mathrm{L})$ and counterstained with a 1:1:1 mixture of APC-conjugated anti-CD9, anti-CD63, and/or anti-CD81 Pan Tetraspanin detection antibodies (supplied in the kit, $4 \mu \mathrm{L}$ each) in a total volume of $135 \mu \mathrm{L}$. The plate was incubated at $450 \mathrm{RPM}$ for $1 \mathrm{~h}$ at room temperature. Next, the samples were washed twice in PBS and resuspended in MACSPlex buffer $(150 \mu \mathrm{L})$. Samples were then transferred to a V-bottom 96-well microtiter plate (Thermo Scientific) and analyzed by flow cytometry using a MACSQuant Analyzer 10 flow cytometer (Miltenyi Biotec). Flowjo software (version 10.6.2, Flowjo, LLC) was used to analyze flow cytometric data. Median fluorescence intensities (MFI) for capture bead subsets were background-corrected by subtracting respective MFI values from matched non-EV containing buffer controls that were treated exactly like EV samples (buffer + capture beads + antibodies). All incubation steps were performed in the dark. The characterization results are presented in Figure S2, Supporting Information.

FL Analysis Setup: The fluorescence (FL) measurements on the engineered $\mathrm{mNG}-\mathrm{EVs}$ were performed with the 100X oil immersion lens of a Zeiss inverted microscope Axio Observer Zl, equipped with a thermoelectrically cooled CCD camera (Andor iXon3 $888 \mathrm{EMCCD}, 100^{\circ} \mathrm{C}$ ). The microscope included a $455 \mathrm{~nm}$ centered LED and a GFP-1828A-000 filter set (Semrock) for excitation of the mNG-CD63 protein. The images were acquired with the following settings: $1 \mathrm{MHz}$ at 16 bit, EMG20.

The FL measurements on the wt-EVs were performed with a $100 \times$ oil immersion lens of a Zeiss inverted microscope Colibri 5, equipped with a Hamamatsu CCD Camera (Orca Flash 4). The microscope was equipped with four LEDs, centered at 385, 475, 555, and $630 \mathrm{~nm}$, respectively. All LEDs included individual excitation filters for the correct fluorophore excitation and minimal crosstalk (Figure S1, Supporting Information). The images were acquired with the following settings: 60\% Fieldstop and $30 \%$ Aperture. The 475,555 , and $630 \mathrm{~nm}$ LEDs were set at $100 \%$ power, whereas the $385 \mathrm{~nm}$ LED was set at $60 \%$ power to reduce image background and noise.

All the images, for both engineered and wild type EVs, were acquired using $2 \mathrm{~s}$ acquisition time. All the results were collected for the EVs included in a microscope area ( $133.12 \mu \mathrm{m} \times 133.12 \mu \mathrm{m}$ for $100 \times$ objective lenses).

AFM Setup: The AFM measurements were performed in liquid conditions on a JPK AFM system (NanoWizard 3), under Quantitative Imaging (QI) Mode. QI is a force spectroscopy-based imaging mode that records a complete force distance curve at each pixel of the image, providing height information, but also adhesion and mechanical properties of the sample, that is, Young Modulus. As there are nearly no lateral forces involved, this imaging mode is particularly suitable for soft samples, such as cells and single particles, that is, EVs. For the measurements, quartz-like NANOSENSORS qp-BioAC-CI AFM probes (CB2 cantilever, $0.1 \mathrm{~N} \mathrm{~m}^{-1}$ force constant) were used.

The scanning parameters, including Setpoint, Z-length, Pixel Time, Z-range, and $\mathrm{N}^{\circ}$ of pixels, were optimized for different images, depending on their desired resolution, and were kept constant for the same image types. The threshold height used for EV identification was $15 \mathrm{~nm}$.

Single EV Analysis Platform: Experiments were performed on a combined inverted microscope and AFM setup aligned with the optical axis of the microscope, using $170 \mu \mathrm{m}$ thick coverslips from Ibidi (Gridded Glass Coverslips Grid-50). The coverslips included four imprinted grids for area identification and were further patterned with marks (equidistant groups of four crosses per group, $5 \mu \mathrm{m}$ long and 50-60 nm deep), using a focused ion beam (FIB). This step was necessary for precise area identification and overlap of the FL and AFM images.

sEVs were first covalently captured onto a coverslip inserted in a chamber well, following our previously reported functionalization protocol.[26] Briefly, the coverslip was first cleaned in a 5:1:1 solution of DI water, $\mathrm{H}_{2} \mathrm{O}_{2}$, and $\mathrm{NH}_{4} \mathrm{OH}\left(88{ }^{\circ} \mathrm{C}, 10 \mathrm{~min}\right)$ and activated with (3-aminopropyl)triethoxysilane (APTES, $5 \% \mathrm{v} / \mathrm{v}$ in $95 \%$ ethanol, $10 \mathrm{~min}$ ) and glutaraldehyde (GA, $1 \% \mathrm{v} / \mathrm{v}$ in $1 \mathrm{x}$ PBS, $1 \mathrm{~h}$ ). Thereafter, EVs were covalently immobilized on the GA surface for $1 \mathrm{~h}$. For the engineered mNG-EV measurements, this step was followed by PBS washing, which completed the functionalization. For the wild type EVs instead, the EV capture step was followed by deactivation of the remaining active groups with tris-ethanolamine (Tris-ETHA, $0.1 \mathrm{M}$ Tris buffer and $50 \mathrm{~mm}$ ethanolamine, $\mathrm{pH} 9.0,30 \mathrm{~min})$ and casein $(0.05 \% \mathrm{w} / \mathrm{v}$ in $1 \times \mathrm{PBS}, 90 \mathrm{~min})$ in order to minimize non-specific binding (NSB) of the fluorescently labelled antibodies. Finally, the coverslips with immobilized sEVs were incubated with a solution containing the three fluorescently labelled CD81-APC, CD63-R-PE, and CD9-PB antibodies (2 nM each in 1× PBS, for $2 \mathrm{~h}$ ) and washed with $1 \times$ PBS for the subsequent analyses. Being the EVs immobilized at a concentration of $0.33 \mathrm{pm}$, they were incubated with an antibody concentration more than 6000 -fold in excess to guarantee proper tetraspanin staining. All the $\mathrm{FL}$ and AFM measurements were performed in liquid, in $1 \times$ PBS.

Following functionalization, the chamber well was positioned on top of the inverted microscope and the AFM tip on top was then aligned with the microscope's optical axis, in order to scan the same fluorescently imaged area. After alignment, FL images with the different LEDs were taken, followed by AFM scanning. The presence of the FIB marks on the coverslips was essential in this phase, as it could overcome for small shifts between the FL and AFM scans, occurring although the initial tip-lens alignment step, and enabled the accuracy of the combined measurements.

The AFM scans were first taken on top of the FIB marks for big spots $(30 \mu \mathrm{m} \times 30 \mu \mathrm{m})$ at low resolutions, for area identification, and then for small spots $(5 \mu \mathrm{m} \times 5 \mu \mathrm{m})$ at high resolution $(256 \times 256$ pixels, $19.5 \mathrm{~nm}$ pixel resolution), for accurate EV size determination.

FL Image Processing and Analysis: The FL images were processed and analyzed using the Zeiss Image Analysis Software (ZEN, Blue Edition). Briefly, after background generation and subtraction, the "Image overlay" processing function was used to stack the FL images of the different channels (different antibodies) together. Thereafter, a custom analysis workflow (Analysis window-segment region classes independently method) containing appropriate values of intensity threshold, smoothing, dot separation, and area for each channel was created and applied to the stacked image. For each fluorescent spot, different parameters including the integrated intensity, pixel count, etc. were extracted and analyzed. The same workflow was applied for the three different cell-line derived sEVs.

A custom-made MATLAB script was utilized to consolidate the data files generated by the ZEN software for each fluorescence channel, to calculate the total numbers of EVs detected and classify the EVs based on the coexistence of various markers.

AFM Image Processing and Analysis: The AFM height images were processed using the JPK data processing software. First, a custom workflow containing plane/line fit corrections and a median filter was applied to all images. This allowed the removal of noise and artifacts coming from substrate tilting. Following correction, Gwyddeon software and a MATLAB code were used for analysis. In particular, a maximum height threshold of $15 \mathrm{~nm}$ was set for EV identification and the surface area of all the particles above threshold was extracted and analyzed. Assuming surface area conservation, the EV equivalent diameters were then obtained for single vesicles.

The AFM YM images were processed using the JPK data processing software. In particular, a custom workflow containing baseline subtraction, contact point determination, vertical tip position calculation, and elasticity fit was applied to all the force curve files of the scanned areas. Following correction, the YM image data were analyzed using a custom MATLAB code. In particular, a mask based on each AFM Height image, where the EVs were identified, was generated using Gwyddeon. The mask was then transferred to the corresponding AFM YM image and the mean $Y M$ for each vesicle was extracted. Finally, a script in MATLAB was used to connect each EV to its mean YM value.

tSNE Analysis: For an easier overlook of the acquired data, the FL integrated intensities of the three analyzed tetraspanins of the EVs were mapped onto a 2D plane using tSNE. Different tSNE mappings with perplexity values ranging from 1-40 were tested. The optimal value of perplexity was determined by investigating each perplexities' pseudo-BIC value. ${ }^{[4]}$ The maximum number of iterations in the algorithm was kept sufficiently large to ensure convergence. As the parameters were 
continuous, the tSNE algorithm was run with a standardized Euclidean distance metric. After mapping the data with tSNE, cluster algorithms were applied on the result, making it possible to reveal subpopulations of the vesicles which could be analyzed. The number of clusters was determined by visual inspection of the tSNE plots for each cell-line. Each vesicle was then sorted into one of the identified clusters by applying a linkage hierarchical clustering algorithm. For HEK293 a tSNE mapping with a perplexity of 29 and with five identified clusters was selected, and for $\mathrm{CbMSC}$ a tSNE mapping with a perplexity of 32 and with three identified clusters was selected. For the third cell line, THPI, a tSNE mapping with a perplexity of 17 and with 5 identified clusters was selected. Both the tSNE and the cluster analysis were performed using a MATLAB script.

\section{Supporting Information}

Supporting Information is available from the Wiley Online Library or from the author.

\section{Acknowledgements}

The entire study was supported by a grant from the Erling Persson Family Foundation. A.D., F.P., and S.S.S. acknowledge also the grant funded by the Swedish Research Council (Contract No. 2016-05051). SELA is supported by the Swedish Research Council and SSF-IRC (FormulaEx). The authors acknowledge the work of Lars Riekehr (Uppsala University, Sweden), who patterned the marks needed for precise spot identification on the coverslips used for the combined FL-AFM measurements, using FIB. They also acknowledge Marco Colangelo (MIT, Boston) for the help in the MATLAB coding. Figure 1 was realized using BioRender.com. A.G. is an International Society for Advancement of Cytometry (ISAC) Marylou Ingram Scholar 2019-2023). D.R.M. acknowledges the support from Cihan University-Erbil, in Iraq.

\section{Conflict of Interest}

The authors declare no conflict of interest.

\section{Author Contribution}

S.C. conceptualized the study, performed the combined measurements on the mNG-EVs (together with C.P.) and the measurements on wt-EVs, performed the control measurements, processed, analyzed, and plotted the data, wrote the original manuscript and designed the figures under the supervision of A.D. F.P. conceptualized the study, developed the combined FL-AFM platform, performed the measurements on the stability of the vesicles and on the capture efficiency of the protocol. F.S. extracted the size and YM information from the AFM data, performed the tSNE and cluster analysis on the wt-EVs. A.G. advised on study design, coordinated production, isolation, and characterization of $\mathrm{EVs}$, performed the multiplex bead-based flow cytometry EV surface profiling experiments and analyzed the corresponding data. C.P. performed the FL-AFM combined measurements on the $\mathrm{mNG}-\mathrm{EV}$ s under the supervision of F.P. and S.C. S.S.S. wrote the MATLAB script to consolidate the FL data and classify EVs based on the co-existence of various markers, contributed to the tSNE analysis. D.R.M. contributed to isolate and purify the SEV samples and to characterize them by NTA. D.G. contributed to characterize the sEV samples by NTA and WB. S.E.A. advised on study design and led the lab generating all EVs for the study. J.L. reviewed and edited the manuscript, acquired the funding. A.D. conceptualized the study, analyzed the data, supervised the study, and wrote the original manuscript. All authors reviewed and have given approval to the final version of the manuscript.

\section{Data Availability Statement}

The data that support the findings of this study are available from the corresponding author upon reasonable request.

\section{Keywords}

AFM, extracellular vesicles, fluorescence microscopy, mechanical properties, protein profiling, single vesicle profiling, size profiling

Received: December 28, 2020

Published online: March 7, 2021

[1] D. E. Murphy, O. G. De Jong, M. Brouwer, M. J. Wood, G. Lavieu, R. M. Schiffelers, P. Vader, Exp. Mol. Med. 2019, 51, 32.

[2] A. L. S. Revenfeld, R. Bæk, M. H. Nielsen, A. Stensballe, K. Varming, M. Jørgensen, Clin. Ther. 2014, 36, 830.

[3] P. Vader, X. O. Breakefield, M. J. A. Wood, Trends Mol. Med. 2014, 20, 385

[4] S. El Andaloussi, I. Mäger, X. O. Breakefield, M. J. A. Wood, Nat. Rev. Drug Discovery 2013, 12, 347.

[5] E. Willms, H. J. Johansson, I. Mäger, Y. Lee, K. E. M. Blomberg, M. Sadik, A. Alaarg, C. I. E. Smith, J. Lehtiö, S. El Andaloussi, M. J. A. Wood, P. Vader, Sci. Rep. 2016, 6, 22519.

[6] L. Margolis, Y. Sadovsky, PLoS Biol. 2019, 17, e3000363.

[7] C. Lässer, S. Chul, L. Jan, Mol. Aspects Med. 2018, 60, 1.

[8] D. K. Jeppesen, A. M. Fenix, J. L. Franklin, J. N. Higginbotham, Q. Zhang, L. J. Zimmerman, D. C. Liebler, J. Ping, Q. Liu, R. Evans, W. H. Fissell, J. G. Patton, L. H. Rome, D. T. Burnette, R. J. Coffey, Cell 2019, 177, 428.

[9] T. L. Whiteside, Expert Rev. Mol. Diagn. 2015, 15, 1293.

[10] S. L. N. Maas, X. O. Breakefield, A. M. Weaver, M. G. Hospital, Trends Cell Biol. 2017, 27, 172.

[11] T. A. Hartjes, S. Mytnyk, G. W. Jenster, V. Van Steijn, Bioengineering 2019, 6, 7 .

[12] K. Lee, K. Fraser, B. Ghaddar, K. Yang, E. Kim, L. Balaj, E. A. Chiocca, X. O. Breake, H. Lee, R. Weissleder, ACS Nano 2018, 12, 494.

[13] Y. Tian, M. Gong, G. Su, S. Zhu, W. Zhang, S. Wang, Z. Li, C. Chen, L. Li, L. Wu, X. Yan, ACS Nano 2018, 12, 671.

[14] G. G. Daaboul, P. Gagni, L. Benussi, P. Bettotti, M. Ciani, M. Cretich, D. S. Freedman, R. Ghidoni, A. Y. Ozkumur, C. Piotto, D. Prosperi, B. Santini, M. S. Ünlü, M. Chiari, Sci. Rep. 2016, 6, 37246.

[15] R. P. Carney, S. Hazari, M. Colquhoun, D. Tran, B. Hwang, M. S. Mulligan, J. D. Bryers, E. Girda, G. S. Leiserowitz, Z. J. Smith, K. S. Lam, Anal. Chem. 2017, 89, 5357.

[16] D. Wu, J. Yan, X. Shen, Y. Sun, M. Thulin, Y. Cai, L. Wik, Q. Shen, J. Oelrich, X. Qian, K. L. Dubois, K. G. Ronquist, M. Nilsson, U. Landegren, M. Kamali-Moghaddam, Nat. Commun. 2019, 10, 3854.

[17] G. Corso, W. Heusermann, D. Trojer, A. Görgens, E. Steib, J. Voshol, A. Graff, C. Genoud, Y. Lee, J. Hean, J. Z. Nordin, O. P. B. Wiklander, S. El Andaloussi, N. Meisner-Kober, J. Extracell. Vesicles 2019, 8, 1663043.

[18] A. Görgens, M. Bremer, R. Ferrer-Tur, F. Murke, T. Tertel, P. A. Horn, S. Thalmann, J. A. Welsh, C. Probst, C. Guerin, C. M. Boulanger, J. C. Jones, H. Hanenberg, U. Erdbruegger, J. Lannigan, F. L. Ricklefs, S. El Andaloussi, B. Giebel, J. Extracell. Vesicles 2019, 8, 1587567.

[19] F. A. W. Coumans, A. R. Brisson, E. I. Buzas, F. Dignat-George, E. E. E. Drees, S. El Andaloussi, C. Emanueli, A. Gasecka, A. Hendrix, A. F. Hill, R. Lacroix, Y. Lee, T. G. Van Leeuwen, N. Mackman, I. Mäger, J. P. Nolan, E. Van Der Pol, D. M. Pegtel, S. Sahoo, P. R. M. Siljander, G. Sturk, O. De Wever, R. Nieuwland, Circ. Res. 2017, 120, 1632. 
[20] M. Yáñez-Mó, P. R.-M. Siljander, Z. Andreu, A. B. Zavec F. E. Borràs, E. I. Buzas, K. Buzas, E. Casal, F. Cappello, J. Carvalho, E. Colás, A. Cordeiro-da Silva, S. Fais, J. M. Falcon-Perez, I. M. Ghobrial, B. Giebel, M. Gimona, M. Graner, I. Gursel, M. Gursel, N. H. H. Heegaard, A. Hendrix, P. Kierulf, K. Kokubun, M. Kosanovic, V. Kralj-Iglic, E.-M. Krämer-Albers, S. Laitinen, C. Lässer, T. Lener, J. Extracell. Vesicles 2015, 4, 27066.

[21] D. Vorselen, S. M. Van Dommelen, R. Sorkin, M. C. Piontek, J. Schiller, S. T. Döpp, S. A. A. Kooijmans, B. A. Van Oirschot, B. A. Versluijs, M. B. Bierings, R. Van Wijk, R. M. Schiffelers, G. J. L. Wuite, W. H. Roos, Nat. Commun. 2018, 9, 4960.

[22] R. Sorkin, R. Huisjes, F. Boškovic, D. Vorselen, S. Pignatelli, Y. Ofirbirin, J. K. F. Leal, J. Schiller, D. Mullick, W. H. Roos, G. Bosman, N. Regev-rudzki, R. M. Schiffelers, G. J. L. Wuite, Small 2018, 14, 1801650.

[23] L. Zhang, Q. Feng, J. Wang, S. Zhang, B. Ding, Y. Wei, M. Dong, J. Ryu, T. Yoon, X. Shi, J. Sun, X. Jiang, ACS Nano 2015, 9, 9912.

[24] E. Van der Pol, F. A. Coumans, A. Grootemaat, C. Gardiner, I. Sargent, P. Harrison, A. Sturk, T. Van Leeuwen, R. Nieuwland, J. Thromb. Haemostasis 2014, 12, 1182.

[25] S. Sharma, M. Leclaire, J. K. Gimzewski, Nanotechnology 2018, 29 , 132001.

[26] S. Cavallaro, J. Horak, P. Hååg, D. Gupta, C. Stiller, S. S. Sahu, A. Görgens, H. K. Gatty, K. Viktorsson, S. El Andaloussi, R. Lewensohn, A. E. Karlstrom, J. Linnros, A. Dev, ACS Sens. 2019, 4, 1399.

[27] O. P. B. Wiklander, R. B. Bostancioglu, J. A. Welsh, A. M. Zickler, F. Murke, G. Corso, U. Felldin, D. W. Hagey, B. Evertsson, X. M. Liang, M. O. Gustafsson, D. K. Mohammad, C. Wiek, H. Hanenberg, M. Bremer, D. Gupta, M. Björnstedt, B. Giebel, J. Z. Nordin, J. C. Jones, S. El Andaloussi, A. Görgens, Front. Immunol. 2018, 9, 01326.
[28] M. Reth, Nat. Immunol. 2013, 14, 765.

[29] J. Syed, J. Ashton, J. Joseph, G. N. Jones, C. Slater, A. Sharpe, G. Ashton, W. Howat, R. Byers, H. K. Angell, Immunother.: Open Access 2019, 5, 1000157.

[30] G. Corso, I. Mäger, Y. Lee, A. Görgens, J. Bultema, B. Giebel, M. J. A. Wood, J. Z. Nordin, S. El Andaloussi, Sci. Rep. 2017, 7, 11561.

[31] J. Z. Nordin, R. B. Bostancioglu, G. Corso, S. El Andaloussi, Target Identif. Validation Drug Discovery 2019, 1953, 287.

[32] D. Bachurski, M. Schuldner, P.-H. Nguyen, A. Malz, K. S. Reiners, P. C. Grenzi, F. Babatz, A. C. Schauss, H. P. Hansen, M. Hallek, E. P. von Strandmann, J. Extracell. Vesicles 2019, 8, 1596016.

[33] A. Ridolfi, M. Brucale, C. Montis, L. Caselli, L. Paolini, A. Borup, A. T. Boysen, F. Loria, M. J. C. Van Herwijnen, M. Kleinjan, P. Nejsum, N. Zarovni, M. H. M. Wauben, D. Berti, P. Bergese, F. Valle, Anal. Chem. 2020, 92, 10274.

[34] D.-k. Kim, H. Nishida, S. Y. An, A. K. Shetty, T. J. Bartosh, D. J. Prockop, Proc. Natl. Acad. Sci USA 2016, 113, 170.

[35] Q. Zhang, J. N. Higginbotham, D. K. Jeppesen, Y. Yang, W. Li, T. Mckinley, R. Graves-deal, J. Ping, C. M. Britain, K. A. Dorsett, C. L. Hartman, D. A. Ford, R. M. Allen, K. C. Vickers, Q. Liu, L. Jeffrey, S. L. Bellis, R. J. Coffey, Cell Rep. 2019, 27, 940.

[36] Y. Yang, G. Shen, H. Wang, H. Li, T. Zhang, N. Tao, X. Ding, H. Yu, Proc. Natl. Acad. Sci USA 2018, 115, 10275.

[37] J. Kowal, G. Arras, M. Colombo, M. Jouve, J. P. Morath, B. PrimdalBengtson, F. Dingli, D. Loew, M. Tkach, C. Théry, Proc. Natl. Acad. Sci U. S. A. 2016, 113, E968.

[38] F. K. Fordjour, G. G. Daaboul, S. J. Gould, bioRxiv 2019.

[39] Y. Ji, D. Qi, L. Li, H. Su, X. Li, Y. Luo, B. Sun, F. Zhang, B. Lin, T. Liu, Y. Lu, Proc. Natl. Acad. Sci USA 2019, 116, 5979.

[40] G. Corso, I. Mäger, Y. Lee, A. Görgens, J. Bulte, B. Giebel, M. J. A. Wood, J. Z. Nordin, S. El Andaloussi, Sci. Rep. 2017, 7, 11561.

[41] Y. Cao, L. Wang, arXiv 2017, 1708.03229. 